
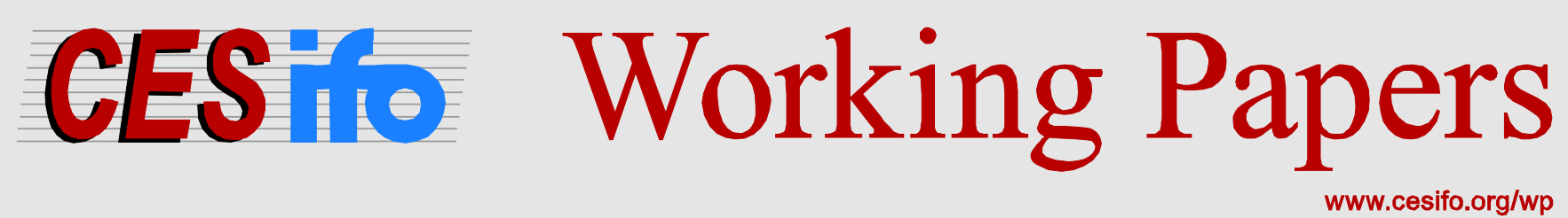

\title{
All-pay-all Aspects of Political Decision Making
}

\author{
Thomas Giebe \\ Paul Schweinzer
}

CESIFO WORKING PAPER NO. 4330

CATEgory 2: Public CHOICE

JULY 2013

An electronic version of the paper may be downloaded

- from the SSRN website:

- from the RePEc website:

- from the CESifo website:

WWw.SSRN.com

www.RePEc.org

www.CESifo-group.org/wp

\section{CESifo}




\title{
All-pay-all Aspects of Political Decision Making
}

\begin{abstract}
We study decision making processes with non-standard all-pay structures. We motivate this interest through a group of regulatory, political, legal, military, and economic applications where individual actions determine the consequences for a larger group or the public. The common features of these examples are a competitive environment, winner-take-all reward structure, and some form of all-pay-all payment rule.
\end{abstract}

JEL-Code: C700, D700, L500.

Keywords: auctions, contests, regulation, conflict.

\author{
Thomas Giebe \\ Microeconomics \\ Technical University Berlin \\ Straße des 17. Juni 135 \\ Germany - 10623 Berlin \\ thomas.giebe@tu-berlin.de
}

\author{
Paul Schweinzer \\ Department of Economics \\ University of York \\ UK - Heslington, York YO10 5DD \\ United Kingdom \\ paul.schweinzer@york.ac.uk
}

Thanks to Bipasa Datta, Alex Gershkov, Arye Hillman, Bettina Klose, Peter Simmons, Jacco Thijssen, and Zaifu Yang for helpful comments. We are grateful for financial support through the University of York Research and Impact Support Fund. 


\section{Introduction}

Many strategic situations can be understood as contests where participants compete for payoffs. Contests have a unifying 'all-pay' property: Participants' efforts or investments are sunk, regardless of whether they win a prize in the competition or not. The most widely studied case is the 'firstprice' payment rule, where players pay their own cost of effort, regardless of winning. Applications include lobbying, sports contests, political competition, labour tournaments, and many other racelike situations. Another popular class of contest applications exhibits a 'second-price' structure, i.e., problems where participants pay the highest losing effort. These are often described as 'wars of attrition,' because the winner only has to match or 'overcome' the best rival's effort in order to win the prize. Typically, the literature models both classes of applications as all-pay auctions or contests, either with an additional element of 'luck' or other influences, or without.

There is recent interest in winner-take-all contests with alternative payment rules, for instance, as arising from the study of legal systems. Our paper adds to this discussion, arguing that there are important applications that fit in the framework of all-pay auctions but are neither captured by the widely studied first-price nor the second-price payment rules. Our contribution is to study two classes of all-pay auction games that we motivate in the following.

The first class of all-pay auctions we study relates to regulatory problems. In these games, the bids are interpreted as proposals, rather than efforts. Consider a regulator who imposes price caps, e.g., the EU commission in the recent case of setting a ceiling on mobile phone roaming prices. ${ }^{1}$ We introduce a 'proposal game' where the regulator asks firms to submit proposals for a new (reduced) price level. The regulator commits to implement the smallest submitted price as a binding price cap for all firms. Thus, we study an all-pay auction where all players pay the winner's bid. We show that such a game is effective in reducing the price level in non-cooperative equilibrium. Importantly, the game does not have unattractive equilibria, in which players propose only minimal reductions. The incentive to propose price reductions is provided by the firm's interest in the competitive advantage of publicity and being perceived as the most consumer friendly. ${ }^{2}$

A related regulatory application is the setting of industry standards. ${ }^{3}$ This problem has previously been modeled as a 'war of attrition' because firms continue to spend resources until a standard emerges. ${ }^{4}$ We consider a 'standard-setting battle,' a regulatory game where the regulator asks firms for proposals and commits to select one of the submitted technologies as the industry standard. Then all other firms have to adopt this standard in the future. In this interpretation, a war of

\footnotetext{
1 The Guardian, Mobile phone firms to be forced to reduce overseas roaming charges, 6-Jul-11.

2 Recent examples for this kind of incentive include Vodafone's announcement of the 'travel promise' (the statement of 3-Aug-2005, starts with 'Commitment to simplify and increase value to Vodafone customers') and T-Mobile's 'Un-carrier event,' both of which are essentially widely advertised announcements of price reductions.

3 The interoperability between competing technologies through the joint adoption of a common standard is usually achieved within standard-setting organisations (SSOs). International examples of SSOs include the International Organisation for Standardisation (ISO) or the International Electrotechnical Commission (IEC). Each individual SSO has its own rules that regulate participation criteria, the methods of evaluation of proposed standards, the voting rules and so on. One of the most common rules is that a patent that applies to the standard must be adopted on 'fair, reasonable, and non-discriminatory terms' (FRAND). For recent surveys, examples, and discussions see, for instance, Geradin (2010) or Organisation for Economic Co-operation and Development (2010).

${ }^{4}$ See, e.g., Bulow and Klemperer (1999).
} 
attrition is inappropriate as development cost of technologies are already sunk at the time of the regulatory game. Then, bids are proposals and payoffs depend on which proposal is implemented. Notice that there is still a payoff to winning in terms of fees from patents or other property rights.

A third application are voting games where the issue at hand is a reduction in bonuses, expenses, etc, of the participating players. Following unfavourable press coverage, for instance, board members might be asked to propose cuts in their annual bonuses. Suppose that shareholders or the board of supervisors commit to implementing the most stringent reduction from among all proposals. It is easy to find many elements of this kind of games such as voluntary reduction proposals, publicity seeking, or payoffs from being the proposer of the implemented proposal in the public press. ${ }^{5}$ Again, these games implement a payoff-reducing outcome in equilibrium where the most stringent proposal is made by the player who values winning the most.

Our second class of all-pay auctions is an extension of the classical war of attrition. Recall that the war of attrition is traditionally motivated by two players (organisms) fighting for a resource. The fight ends as soon as one player gives in, implying that both players pay the loser's effort cost. It is natural to extend this model by taking into account that one player's effort causes a negative externality on the other player, e.g., in the form of injury or destruction (in a military context). Thus, a player's effort choice causes a direct effect in the form of effort cost, and an indirect effect in the form of injury to the rival. This results in both players paying (a function of) total effort. ${ }^{6}$

For both classes of games, we explicitly consider the role of outside options. This is because, depending on the specific application, these may be games within larger games such that the participation decision depends on more than the direct payoff from the auction. For instance, a firm in a regulated industry must abide by the rules, or face fines. Similarly, for a board member, participating in a vote is either obligatory, or absence might be inconsequential in the sense that the vote is binding for that member, anyway.

\section{Literature}

Our paper contributes to the study of all-pay auction games. ${ }^{7}$ Some recent contributions include Krishna and Morgan (1997), Hopkins and Kornienko (2007), Kaplan, Luski, Sela, and Wettstein (2002), and Klose and Kovenock (2011a).

Krishna and Morgan (1997) study a generalised version of the classical war of attrition, as well as the first-price, all-pay auction, with affiliated valuations. They provide revenue ranking results

\footnotetext{
5 Three examples from politics, business, and the public sector, respectively, are: The Hill, House Dem proposes 20 percent pay cut for lawmakers, 10-Apr-2013; Financial Times, BG executive directors to forgo bonuses, 4-Apr2013; The Times, $B B C$ directors agree to take wage cut as pressure grows to reveal celebrity pay, 1-Jul-2010.

${ }^{6}$ This idea is not new. The two-player complete information case of this setup has been recently analysed by Baye, Kovenock, and de Vries (2012). In particular, see their subsection 'Territorial contests with injuries.'

7 The beautiful theory of these games has been developed, among others, by Dasgupta (1986) discussing R\&D races, Hillman and Samet (1987) introducing rent-seeking and lobbying contests, Hillman and Riley (1989) and Baye, Kovenock, and de Vries (1993) analysing competitions for monopoly, Clark and Riis (1998) studying the competition for promotions, and Moldovanu and Sela (2001) discussing optimal prize structures. We hope that the availability of recent and comprehensive surveys of the contests literature such as Garfinkel and Skaperdas (2006), Congleton, Hillman, and Konrad (2007), Corchón (2007), and Konrad (2008) allow us to focus on the discussion of only the most directly related parts of the literature.
} 
for these and other sealed-bid formats. Both Hopkins and Kornienko (2007) and Kaplan, Luski, Sela, and Wettstein (2002) explore non-standard pricing rules which are different from ours, and Klose and Kovenock (2011a) is a study under complete information. Klose and Kovenock (2011b) investigate a setup related to ours - that of identity-dependent externalities-but their analysis and research questions are set within a voting model. Güth and van Damme (1986) and Amann and Leininger (1996) discuss hybrid versions between asymmetric versions of the first- and second-price sealed-bid, all-pay auctions. ${ }^{8}$ In this hybrid format, the prize is still won by the highest bidder but the loser pays her bid while the winner pays a convex combination of her own and the loser's bid. The type of auction we consider relates to contests with externalities; for recent contributions to this field see Maasland and Onderstal (2007) or, again, Klose and Kovenock (2011a).

The papers which are closest to our analysis are Goeree and Turner (2000), Baye, Kovenock, and de Vries (2005), and Baye, Kovenock, and de Vries (2012). The first introduce the all-pay-all auction format - in which all bidders pay a weighted sum of all bids-as the optimal format when all bidders receive some share of the amount of money raised. ${ }^{9}$ Thus, each bidder has an incentive to drive up the price because every participant receives a fixed share of the auction's revenue. In contrast, in our games, players do not benefit from other players' bids.

The paper by Baye, Kovenock, and de Vries (2005) studies litigation systems in a two-player game of incomplete information. They study so called fee-shifting rules which are relevant in the legal context. We study $n$-player games, but for the case of $n=2$ players, our paper is complementary as follows. Our first game corresponds to $(\alpha, \beta, \delta, \theta)=(\alpha, 0,0, \alpha)$ in their notation. ${ }^{10}$ This parameter constellation violates their assumptions $\beta>0$ and (A3) 'internalised legal cost,' which rules out subsidising or taxing of legal expenditures. However, it is equivalent to a legal system where the winner bears its own cost while the loser pays a tax that raises the loser's cost to the level of the winner's cost. This idea is similar in nature to the 'Quayle system,' which attempts to discourage abuse of the court system by compelling the loser to make payments exceeding the loser's own cost. ${ }^{11}$ Our second game corresponds to $(\alpha, \beta, \delta, \theta)=(\alpha, \alpha, \alpha, \alpha)$ if we set $k=1$ (linear payoff) in our model. This constellation is consistent with the assumptions in Baye, Kovenock, and de Vries (2005) for $\alpha=1 / 2$. Thus, within their framework, our paper contributes to the analysis of the legal system ' $(\alpha=1 / 2, \beta=1 / 2)$,' where every party pays one half of total legal expenditure. Moreover, varying our parameters $\alpha$ and $k$ allows us to study (hypothetical) legal systems where litigation costs are subsidised or taxed, compelling the parties to make (potentially nonlinear) payments to the court in addition to (or less than) their actual legal expenditures.

\footnotetext{
${ }^{8}$ For further discussions of asymmetric all-pay auctions see, for instance, Hillman and Riley (1989), Fibich, Gavious, and Sela (2006), Parreiras and Rubinchik (2010), or Szech (2011). For a detailed discussion, we appeal, again, to the above cited survey literature.

${ }^{9}$ A later published variant, Goeree, Maasland, Onderstal, and Turner (2005), is less related to the present work because, there, the payment rules do not exhibit an 'all-pay-all' structure.

10 The parameters $(\alpha, \beta, \delta, \theta)$ are the coefficients of the winner's and loser's linear payoff functions in Baye, Kovenock, and de Vries (2005).

${ }^{11}$ So called after former US Vice President Dan Quayle who chaired a commission which recommended a reform of the American legal system which stipulated the loser to reimburse the winner's legal cost up to the amount actually spent by the loser. The Quayle system is one of the legal systems analysed in Baye, Kovenock, and de Vries (2005).
} 
The present paper fits into the general framework of contests with 'rank-order dependent spillovers' as introduced by Baye, Kovenock, and de Vries (2012). These are contests where players' efforts, in addition to being costly, imply external effects on other players' payoffs. This class of games covers a wide range of well-known problems. While Baye, Kovenock, and de Vries (2012) study two-player games with complete information, we analyse two restricted classes of $n$-player games of incomplete information. Thus, we provide complementary results to theirs. In particular, our first game corresponds to their setup for $(\alpha, \beta, \gamma, \delta, \theta)=(0, \alpha, 0,0, \alpha)$, whereas our second game corresponds to $(\alpha, \beta, \gamma, \delta, \theta)=(\alpha, \alpha, 0, \alpha, \alpha)$ if we set our parameters to $k=1$ (linear payoff) in the second game, and $n=2$ in both games. Thus, in their terminology, our games for $n=2$ exhibit a 'first-order negative direct effect' as well as a 'second-order negative spillover effect,' and our second game, in addition, has a 'first-order negative spillover effect' and a 'second-order negative direct effect.'

Bulow and Klemperer (1999) analyse an incomplete information, dynamic, generalised war of attrition in which players pay some cost before quitting and, possibly, continue to pay a cost even after quitting the game. They interpret the latter 'strategic independence' case as 'standards battle' where all players, actively engaged in promoting a standard or not, pay the costs of not having agreed upon a single standard until such a standard emerges. This payment rule, where every player essentially pays the second-highest bid, is complementary to the one in our first game, where everyone pays the winner's bid. Moreover, we argue that our game provides another application within the problem of standard setting.

Lizzeri and Persico (2000) study uniqueness and existence of equilibria in a general class of auction problems. This class includes mechanisms closely related to the ones we study (see, e.g., their Example 5, a combination of first-price, all-pay auction and the war of attrition). However, their assumption (A7) excludes our setup. It says, i.a., that the loser's payoff must not depend on the winner's bid.

The paper is structured as follows: Following the definition of the environment in the following section, we illustrate the main results through examples. We use the examples to explain how our motivating applications fit the model. We provide the general analysis in 4 . We conclude with a discussion and further applications. The proofs are in the appendix.

\section{The model}

There is a set $\mathcal{N}$ of $n>1$ risk-neutral, ex-ante identical bidders $i \in \mathcal{N}$ who each value winning the game at $\theta_{i}$. These valuations are privately known; all the opponents and the auctioneer know is the symmetric, twice continuously differentiable distribution $F(\theta)_{[0,1]}$, with strictly positive density $f(\theta)=F^{\prime}(\theta)$, from which valuations are drawn. We denote order statistics as follows: The $k^{\text {th }}$ highest of $s$ independent draws from the cumulative distribution function $F$ is $\Theta_{(k: s)}$ and its distribution is denoted by $F^{(k: s)}$. Admissible bids are nonnegative. In case of nonparticipation, a bidder realises the symmetric and commonly known outside option $\bar{u} \in(-\infty, \infty)$. 


\section{Examples}

In this section, we introduce the two auction formats by example, assuming full participation and equilibrium existence. Later, in the general analysis of section 4, we come back to the issues of existence and outside options.

Recall the regulatory 'price cap' game from the introduction. The regulator asks two symmetric duopolists to simultaneously submit a proposal for a new and lower price cap, where the lowest proposed price cap will be implemented. A firm's bid in the auction is measured as the profit reduction implied by the proposal. As argued in the introduction, firms may have a valuation for being seen as proposing the most consumer-friendly regulation. Thus, a firm's payoff from this regulatory game is composed of a positive payoff in the event of winning, minus the profit reduction (bid) proposed by the winning bidder.

Therefore, consider a two-player auction game where the highest bidder wins and both participants pay the highest bid. Taking the existence of a symmetric, monotone bidding function $\beta$ as given, player $i$ of type $\theta_{i} \in[0,1]$ chooses her bid $b$ such as to

$$
\max _{b} \int_{0}^{\beta^{-1}(b)}\left(\theta_{i}-b\right) f\left(\theta_{j}\right) d \theta_{j}-\int_{\beta^{-1}(b)}^{1} \beta\left(\theta_{j}\right) f\left(\theta_{j}\right) d \theta_{j} .
$$

Taking the derivative with respect to $b$, we obtain the first-order condition

$$
f\left(\beta^{-1}(b)\right)\left(\theta_{i}+\beta\left(\beta^{-1}(b)\right)-b\right) \frac{d \beta^{-1}(b)}{d b}-\int_{0}^{\beta^{-1}(b)} f\left(\theta_{j}\right) d \theta_{j}=0
$$

which, after invoking symmetry, $\beta^{-1}(b)=\theta_{i}$ and $\frac{d \beta^{-1}(b)}{d b}=\frac{1}{\beta^{\prime}\left(\theta_{i}\right)}$, reduces to

$$
\beta^{\prime}(\theta)=\frac{f(\theta)}{F(\theta)} \theta
$$

By integrating up both sides over $\left(0, \theta_{i}\right]$ and using the boundary condition $\beta(0)=0$, we obtain the equilibrium bidding function ${ }^{12}$

$$
\int_{0}^{\theta_{i}} \beta^{\prime}(s) d s=\int_{0}^{\theta_{i}} \frac{f(s) s}{F(s)} d s \Longleftrightarrow \beta\left(\theta_{i}\right)=\int_{0}^{\theta_{i}} \frac{f(s) s}{F(s)} d s .
$$

For this example, we restrict attention to the class of distribution functions $F(s)=s^{t}$, for any $t>0$. This includes concave and convex functions. Then $\beta\left(\theta_{i}\right)=t \theta_{i}$, which, for the special case of Uniform valuations, $F(\theta)=\theta$, implies truth-telling, $\beta\left(\theta_{i}\right)=\theta_{i}$. Since there is a value of winning, it is not an equilibrium to propose 'zero reduction' of the price cap. In the litigation framework of Baye, Kovenock, and de Vries (2005), this example corresponds to a legal system, where the winner pays its own legal cost, whereas the loser pays a tax to the court such that legal expenditures are raised to the winner's cost level. ${ }^{13}$ In subsection 4.1 , we extend this example to $n$ players, where every player pays the same multiple of the highest bid.

\footnotetext{
12 Given full participation, this boundary condition is intuitive: If the valuation is zero, then winning has no upside. Thus, the zero type would focus on minimising payments: By making the lowest feasible bid, the bidder minimises the price in the event of winning.

13 The case of taxation is mentioned in Baye, Kovenock, and de Vries (2005) as a way of reducing wasteful legal expenditures.
} 
Now, consider our second class of problems, which extends the idea of the standard war of attrition. Consider a battle between two players where fighting effort is the 'bid.' Producing this effort implies a cost for the player, and, at the same time, it has a direct negative effect on the rival in the form of injury. Thus, a positive effort choice by both players implies that both players pay a function of both bids, while the player who expended the larger effort wins the battle. As a simple example, consider the two-player, all-pay auction where each player pays the average bid. Thus, stipulating full participation and the existence of a symmetric, monotone equilibrium bidding function $\beta$, player $i$ solves

$$
\begin{array}{ll}
\max _{b} & \int_{0}^{\beta^{-1}(b)}\left(\theta_{i}-\frac{b+\beta\left(\theta_{j}\right)}{2}\right) f\left(\theta_{j}\right) d \theta_{j}-\int_{\beta^{-1}(b)}^{1} \frac{b+\beta\left(\theta_{j}\right)}{2} f\left(\theta_{j}\right) d \theta_{j} \Longleftrightarrow \\
\max _{b} & F\left(\beta^{-1}(b)\right) \theta_{i}-\frac{1}{2}\left(b+\int_{0}^{1} \beta\left(\theta_{j}\right) f\left(\theta_{j}\right) d \theta_{j}\right) .
\end{array}
$$

Taking the derivative with respect to $b$, we get

$$
f\left(\beta^{-1}(b)\right) \frac{d \beta^{-1}(b)}{d b} \theta_{i}-\frac{1}{2}=0 .
$$

Again, after invoking symmetry, this reduces to $\beta\left(\theta_{i}\right)^{\prime}=2 f\left(\theta_{i}\right) \theta_{i}$. Clearly, a bidder with zero valuation optimally bids zero. Thus, with boundary condition $\beta(0)=0$, the bid function is

$$
\beta\left(\theta_{i}\right)=2 \int_{0}^{\theta_{i}} f(s) s d s
$$

We have chosen the 'average bid' payment rule, because (7) is equal to (4) in Baye, Kovenock, and de Vries (2005) for their parameters $\alpha=\beta=\delta=\theta=1 / 2$. Thus, our example mechanism corresponds to a legal system where each party pays the average legal expenditure, resulting in twice the actual equilibrium legal expenditure of the American system (in which all litigants pay their own legal expenditures). Baye, Kovenock, and de Vries (2005) show that there is a tradeoff between the American and the British systems (under which the loser pays the own costs and, in addition, reimburses the winner for all legal costs): the American system exhibits higher expected payoffs of litigants (implying incentives in favor of litigation), while the British system results in higher total expected legal expenditures per trial (reducing litigation incentives). On both measures, our example system lies between the American and the British systems, representing a compromise. ${ }^{14}$ In subsection 4.2 , we analyse a more general $n$-player version, where bidders pay a multiple of a (non)linear function of all bids.

\footnotetext{
${ }^{14}$ Baye, Kovenock, and de Vries (2005) show that these performance measures are a function of their $\beta$ only, with $\beta=1$ in the American, and $\beta=0$ in the British system, while our example has $\beta=1 / 2$. Apart from this case, we cannot compare results because as soon as we deviate from $\alpha=1 / 2$ and linear payoffs, we violate assumption (A3) in Baye, Kovenock, and de Vries (2005), which is incorporated in their solution from the very beginning. Moreover, note that these results of Baye, Kovenock, and de Vries (2005) are comparable with ours only given that there is litigation, i.e., participation.
} 


\section{Analysis}

\subsection{Everyone pays (a multiple of) the highest bid}

In the following, we analyse the all-pay auction game where all bidders pay the same constant multiple $\alpha>0$ of the highest bid. We study the class of symmetric, pure-strategy equilibria with continuous, strictly monotone bid functions conditional on participation. In the unique equilibrium of this class, bidders participate (and make positive bids) if their valuations exceed a cutoff value, denoted by $\hat{\theta}$. This cutoff depends on the common outside option $\bar{u}$ and implies full (resp. no) participation for sufficiently low (resp. high) outside options. Unfortunately, this cutoff value cannot be given in closed form, so we define it implicitly, using the equilibrium interim expected payoff of a bidder with valuation $\theta_{i} \geq \hat{\theta}$, denoted by $\Pi\left(\hat{\theta}, \theta_{i}\right)$ as follows. Our first result pins down a boundary condition for the sought bidding function.

Lemma 1. In any symmetric, monotone, pure-strategy equilibrium of the specified all-pay auction games, $\beta(\hat{\theta})=0$.

Proposition 1. Consider the all-pay auction with symmetric outside options, $\bar{u} \in(-\infty, \infty)$, where all bidders pay the multiple $\alpha>0$ of the highest bid. This auction game possesses an equilibrium that is unique in the class of symmetric, pure-strategy equilibria with continuous and strictly monotone bid functions as follows. Define, for $\theta_{i} \geq \hat{\theta}$,

$$
\Pi\left(\hat{\theta}, \theta_{i}\right)=1-(n-1) \int_{\hat{\theta}}^{1} \frac{f(t) t}{F(t)} d t-\int_{\theta_{i}}^{1} F^{n-1}(t) d t .
$$

In equilibrium, there is full participation if $\bar{u} \leq \Pi(0,0)$ and no participation if $\bar{u} \geq \Pi(1,1)=1$. Otherwise, the cutoff valuation $\hat{\theta}$, above which a player participates, is implicitly defined by $\bar{u}=$ $\Pi(\hat{\theta}, \hat{\theta})$. The equilibrium bidding function is, for participating bidders $\theta_{i} \geq \hat{\theta}$, defined as

$$
\beta\left(\theta_{i}, \bar{u}\right)=\frac{n-1}{\alpha} \int_{\hat{\theta}}^{\theta_{i}} \frac{s f(s)}{F(s)} d s,
$$

where $\hat{\theta}=0$ if $\bar{u} \leq \Pi(0,0)$.

The monotone bid function implies allocative efficiency in the usual sense that the highest type wins. Observe that a player's interim expected equilibrium payoff (8) is independent of $\alpha$. In this sense, the exact payment rule is immaterial within the class of rules we study. Thus, by varying $\alpha$ we can manipulate the size of equilibrium bids. This includes over- as well as underbidding one's valuation in equilibrium, and, interestingly, also 'truthful bidding' for all types, which we can show for a specific class of distribution functions: Suppose that $F(\theta)=\theta^{t}$ for some $t>0$ then, in the symmetric equilibrium, all players bid their valuations, $\beta\left(\theta_{i}\right)=\theta_{i}$ for sufficiently small outside options, $\bar{u} \leq-\alpha^{2} /(1+\alpha)$, and $\alpha$ and $n$ are such that $\alpha /(n-1)=t$. In order to see this, set $F(s)=s^{\alpha /(n-1)}$, to get $s f(s) / F(s)=\alpha /(n-1)$. Then (9) straightforwardly simplifies to $\beta\left(\theta_{i}, \bar{u}\right)=\theta_{i}{ }^{15}$

\footnotetext{
${ }^{15}$ Similarly, replace $t f(t) / F(t)$ in (8) by $\alpha /(n-1)$, simplify, and solve $\bar{u}=\Pi(0,0)$ to obtain the required upper bound of the outside option.
} 
In the literature section we identify an overlap with the model of Baye, Kovenock, and de Vries (2005). However, we cannot compare results because we violate their assumption (A3) on which their relevant results rest. Note that-being exogenous $-\alpha$ can be a function of $n$, leaving room for other interpretations and applications. For instance, consider a proposal game where only the winning proposal is implemented and costly. Then $\alpha=1 / n$ implies that all bidders pay an equal share of the cost of the implemented proposal.

Observe that the game does not have an equilibrium where all players bid zero, except for the trivial case where the outside option is more valuable than winning the game, $\bar{u} \geq 1$. Moreover, the result extends to asymmetric outside options as long as they are commonly known to be suffiently low to ensure full participation.

\subsection{The price as a function of all bids}

We now turn to the second set of applications, modelled as an all-pay-all auction where all bidders pay the multiple $\alpha>0$ of (a function of) all bids. Players obviously do not participate if their outside option exceeds $\bar{u}=1$, while for sufficiently low outside option, there is full participation. Otherwise, in every symmetric, monotone, pure-strategy bidding equilibrium, there exists a cutoff value, denoted by $\hat{\theta}$, above which a player-type participates in the auction. Unfortunately, this cutoff value cannot be given in closed form, so we define it implicitly, using the equilibrium interim expected payoff of a bidder with valuation $\theta_{i} \geq \hat{\theta}$, denoted by $\Pi\left(\hat{\theta}, \theta_{i}\right)$, as follows.

Proposition 2. Consider the all-pay auction with symmetric outside options, $\bar{u} \in(-\infty, \infty)$ where every bidder pays a function of all bids, $\alpha \sum_{j=1}^{n}\left(b_{j}\right)^{k}$, with $\alpha, k>0$. This auction game possesses an equilibrium that is unique in the class of symmetric, continuous, monotone, and pure-strategy equilibria. Define, for $\theta_{i} \geq \hat{\theta}$,

$$
\Pi\left(\hat{\theta}, \theta_{i}\right)=F^{n-1}(\hat{\theta}) \hat{\theta}+\int_{\hat{\theta}}^{\theta_{i}} F^{n-1}(s) d s-(n-1) \int_{\hat{\theta}}^{1}(1-F(s))\left(F^{n-1}(s)\right)^{\prime} s d s .
$$

In equilibrium, there is full participation if $\bar{u} \leq \Pi(0,0)$ and no participation if $\bar{u} \geq \Pi(1,1)=1$. Otherwise, the cutoff valuation $\hat{\theta}$, above which a player participates, is implicitly defined by $\bar{u}=$ $\Pi(\hat{\theta}, \hat{\theta})$. The equilibrium bidding function is, for participating bidders $\theta_{i} \geq \hat{\theta}$, defined as

$$
\beta\left(\theta_{i}, \bar{u}\right)=\left(\frac{1}{\alpha} \int_{\hat{\theta}}^{\theta_{i}}\left(F^{n-1}(s)\right)^{\prime} s d s\right)^{\frac{1}{k}}
$$

where $\hat{\theta}=0$ if $\bar{u} \leq \Pi(0,0)$.

As in the previous subsection, this equilibrium implies allocative efficiency and the interim expected payoffs, (10), are independent of the parameters $\alpha$ and $k$. Thus, the specific payment rule (within the class we study) affects the size of equilibrium bids, but leaves expected payoffs unchanged. Again, by changing $\alpha$ and $k$ we can implement over-, and underbidding of the players' 
valuations. We can also show examples of truthful bidding in equilibrium. ${ }^{16}$

In terms of novelty, apart from the example presented in section 3, our result for $n=2$ is outside of the 'fee-shifting' litigation mechanisms studied by Baye, Kovenock, and de Vries (2005), implying subsidisation or taxation of legal expenditures in a (non)linear way.

Similar to the statement in the previous subsection, we mention that the auction studied here does not have an equilibrium where all players bid zero, and the result also extends to asymmetric outside options as long as they are known to be sufficiently low. Finally, the parameters $\alpha$ and $k$, being exogenous, can be functions of $n$, leaving room for more applications and interpretations.

\section{Concluding discussion}

The present study contributes to the analysis of all-pay auctions with payment rules that differ from the classical first- and second-price rules. We provide results that are complementary to recent studies covering applications like litigation, standards wars, or battles with injuries or destruction. In particular, the latter seems to be a natural modification of the classical war of attrition.

Moreover, our contribution is normative in the sense that we introduce proposal games that, in principle, might find application in a regulatory setting, or, similarly, in situations where a body or group of decision makers can be obliged to propose reductions of their own payoffs. The central idea is that the members of such groups (or industries) may value to be known as the proposer of the adopted bill or measure.

A policy implication is that, if a reduction of the decision makers' income increases welfare, then transparency, publicity, and a reward of the most aggressive proposal can be utilised to achieve a welfare improvement even though the decision is a result of independent utility maximisation by the participants. Moreover, in a regulatory setting, it might be an advantage if proposals are made by the firms, who are better informed about cost structures and their willingness to forgo short-term profits for an increase in popularity and market share.

For both classes of games we find similar results. The games possess monotone equilibria implying efficiency (with respect to publicity). They do not have unattractive equilibria with zero bids, making collusion harder. The specific payment rule does not affect interim payoffs, but it affects the size of bids. This implies that the designer, in order to affect payoffs, can concentrate on other design aspects, like the size of outside options or the number of players.

We consider the games we study to be games within larger games, as, e.g., in the case of litigation or a board room vote. There, players might be willing to accept negative payoffs in equilibrium

${ }^{16}$ Consider full participation and $F(\theta)=\theta^{t}$, for some $t>0$, and the auction parameters are $k=t(n-1)+1$ and $\alpha=(k-1) / k$, then the equilibrium exhibits truth-telling $\beta(\theta)=\theta$ : Inserting $F(s)=s^{t}$ into (11) and setting the term equal to $\theta$ (truthful bidding), we get

$$
\theta=\left(\frac{1}{\alpha} \int_{0}^{\theta}\left(s^{t(n-1)}\right){ }^{\prime} s d s\right)^{\frac{1}{k}} \Rightarrow \alpha \theta^{k}=\frac{t(n-1)}{t(n-1)+1} \theta^{t(n-1)+1} .
$$

This equation is satisfied if we insert the proposed $\alpha=(k-1) / k$ and $k=t(n-1)+1$. This result is nongeneric in the sense that it relies on a special distributional assumptions. 
because nonparticipation would imply quitting a larger, presumably overall profitable, game. We take this into account by allowing for arbitrarily large negative or positive outside options. ${ }^{17}$

Our study leaves room for many relevant extensions. Depending on the application, the independent private values assumption might not be appropriate. Our regulatory proposal game is stylised and needs to be analysed within a full-fledged oligopoly game. The issue of collusion might be important. The classes of payment rules we study as well as the symmetric players assumption is restrictive. ${ }^{18}$ For some applications, a sequential modelling version might be more appropriate. Finally, experimental testing of our results may reveal a better understanding of their applicability.

We have motivated our study with two leading applications, the regulatory proposal game and the war of attrition with injuries. In addition, we have shown that special cases of our games are relevant within the frameworks of previous studies on litigation. There is a host of further applications that are related to the payment rules we study but fit our model less closely: 'races to the bottom,' (international) tax competition, ${ }^{19}$ officials proposing a range of measures after an expenses scandal, the social game of 'keeping up with the Joneses,' or the 'filibuster' where the politician with the most stamina succeeds and every participant suffers the participants' combined effort costs (e.g., in terms of time or delay, or, a loss of public esteem for all politicians). In another group of applications, the payment rule is interpreted as a collective punishment by a third party proportional to (and in retaliation for) attacks on that party, where the attackers are motivated by acclaim for the most daring or successful attack. There, we have in mind the conflict between Hamas/Fatah and Israel, or the case of the Somali pirates whose attacks on merchant ships became ever more daring before the international community decided to (successfully) punish these attacks.

\section{Appendix}

Proof of Lemma 1. We need to show that we can rule out symmetric, pure-strategy bid functions that prescribe a strictly positive bid for the lowest participating type $\hat{\theta}$, say $b_{0}>0$ plus a strictly monotone, type-dependent, part. In order to see this, consider first the case of full participation. Then, under a monotone bidding function, a bidder with value between zero and $b_{0}$ bids more than $b_{0}$ and wins with positive probability. In case of winning, she would have to pay more than her value, while in case of losing her payoff is independent of her own bid. Thus, she can strictly improve her payoff by reducing her bid, contradicting the candidate.

Second, consider the case of partial participation and focus on the indifferent bidder with valuation $\theta_{i}=\hat{\theta}$. With positive probability, the indifferent bidder is the only bidder in the auction. Then he wins with certainty and his payoff is decreasing in his bid. Thus, a smaller bid is better. On the other hand, if there are other active bidders, then, by the definition of being the indifferent bidder, these other bidders have higher values and bid higher. Then the indifferent bidder never wins and his payoff is independent of his bid. Therefore, there is an incentive to reduce the bid. We conclude

\footnotetext{
17 Negative equilibrium payoffs are discussed, e.g., in Krishna and Morgan (1997).

18 Relaxing the symmetry assumption within our framework seems elusive at this time.

19 For references see, for example http://en.wikipedia.org/wiki/Race_to_the_bottom.
} 
that every strictly monotone bid function must satisfy $\beta(\hat{\theta})=0$.

Proof of Proposition 1. If $\bar{u} \geq 1$, the outside option is superior to the auction. Thus, we restrict attention to $\bar{u} \in(-\infty, 1)$. We moreover focus on symmetric, pure-strategy equilibria with continuous, strictly monotone bid functions. Therefore, participation is governed by a comparison between the common outside option and the equilibrium expected payoff from participation. This implies a common cutoff valuation, denoted by $\hat{\theta} \in[0,1]$, below which a player prefers the outside option. This includes the cases of 'full' and 'no' participation. From Lemma 1 , we know that $\beta(\hat{\theta})=0$.

Suppose that there is an equilibrium where all bidders take their outside options if their valuations are below the common threshold value $\hat{\theta}$. If their valuations exceed this value, then they bid according to the strictly monotonic bidding function $\beta$. We first determine this bidding function, taking the threshold value $\hat{\theta} \in[0,1]$ as given. We start by considering bidder $i$ 's decision problem when her value exceeds the threshold value, $\theta_{i}>\hat{\theta}$. W.I.o.g. we only consider $i$ 's positive bids $\beta(z)$ for $z \in(\hat{\theta}, 1]$. Bidder $i$ 's expected profit from bidding $\beta(z)$, denoted by $\pi\left(z, \theta_{i}\right)$, is

$$
\begin{aligned}
\pi\left(z, \theta_{i}\right) & =F^{(1: n-1)}(z)\left(\theta_{i}-\alpha \beta(z)\right)-\left(1-F^{(1: n-1)}(z)\right) \mathbb{E}\left[\alpha \beta\left(\Theta_{(1: n-1)} \mid \Theta_{(1: n-1)}>z\right]\right. \\
& =F^{n-1}(z)\left(\theta_{i}-\alpha \beta(z)\right)-\int_{z}^{1} \alpha \beta(s) d F^{n-1}(s) ; \\
\frac{\partial \pi\left(z, \theta_{i}\right)}{\partial z} & \left.=\left(F^{n-1}(z)\right)^{\prime}\left(\theta_{i}-\alpha \beta(z)\right)-F^{n-1}(z) \alpha \beta^{\prime}(z)\right)+\alpha \beta(z)\left(F^{n-1}(z)\right)^{\prime}=0 \\
\Longleftrightarrow \beta^{\prime}(z) & =\frac{1}{\alpha} \frac{\left(F^{n-1}(z)\right)^{\prime}}{F^{n-1}(z)} \theta_{i}=\frac{(n-1)}{\alpha} \frac{F(z)^{n-2}(z) f(z)}{F^{n-1}(z)} \theta_{i}=\frac{(n-1)}{\alpha} \frac{f(z)}{F(z)} \theta_{i} .
\end{aligned}
$$

As argued above, $\beta(\hat{\theta})=0$. Using this as the boundary condition, our equilibrium bid function is found by integrating the above differential equation, with one exception: Due to $F(0)=0$, the differential equation is not defined at $\hat{\theta}=0$. In that case, we integrate over the open interval $\left(0, \theta_{i}\right]$ and define $\beta(0)=0$, corresponding to $\beta(\hat{\theta})=0$. In symmetric equilibrium, $z=\theta_{i}$. Thus,

$$
\beta\left(\theta_{i}\right)=\frac{(n-1)}{\alpha} \int_{\hat{\theta}}^{\theta_{i}} \frac{f(s) s}{F(s)} d s .
$$

This corresponds to (9). Note that, at $s=0$, the numerator as well as the denominator vanish. Thus, the integrand is bounded by l'Hôspital's rule,

$$
\lim _{s \rightarrow 0} \frac{f(s) s}{F(s)}=\lim _{s \rightarrow 0} \frac{f^{\prime}(s) s}{f(s)}+1=1,
$$

and, therefore, the integrand of (14) is bounded everywhere. Since every bounded function can be integrated, the resulting bidding function is well defined.

We now turn to $i$ 's expected equilibrium profit, denoted by $\Pi\left(\hat{\theta}, \theta_{i}\right)$, where all players follow (14) and $\theta_{i}>\hat{\theta}$.

$$
\begin{aligned}
\Pi\left(\hat{\theta}, \theta_{i}\right) & =F^{n-1}\left(\theta_{i}\right)\left(\theta_{i}-\alpha \beta\left(\theta_{i}\right)\right)-\int_{\theta_{i}}^{1} \alpha \beta(s) d F^{n-1}(s) \\
& =F^{n-1}\left(\theta_{i}\right)\left(\theta_{i}-(n-1) \int_{\hat{\theta}}^{\theta_{i}} \frac{f(t) t}{F(t)} d t\right)-\underbrace{\int_{\theta_{i}}^{1}(n-1) \int_{\hat{\theta}}^{s} \frac{f(t) t}{F(t)} d t\left(F^{n-1}(s)\right)^{\prime} d s}_{=:(A)} .
\end{aligned}
$$


Interchanging the order of integration, the last term can be written as

$$
\begin{aligned}
(A) & =\int_{\hat{\theta}}^{\theta_{i}} \int_{\theta_{i}}^{1}\left(F^{n-1}(s)\right)^{\prime} d s(n-1) \frac{f(t) t}{F(t)} d t+\int_{\theta_{i}}^{1} \int_{t}^{1}\left(F^{n-1}(s)\right)^{\prime} d s(n-1) \frac{f(t) t}{F(t)} d t \\
& =\int_{\hat{\theta}}^{\theta_{i}}\left(1-F^{n-1}\left(\theta_{i}\right)\right)(n-1) \frac{f(t) t}{F(t)} d t+\int_{\theta_{i}}^{1}\left(1-F^{n-1}(t)\right)(n-1) \frac{f(t) t}{F(t)} d t .
\end{aligned}
$$

After reinserting $(A)$ into (16) and straightforward simplification, we obtain

$$
\Pi\left(\hat{\theta}, \theta_{i}\right)=F^{n-1}\left(\theta_{i}\right) \theta_{i}-(n-1) \int_{\hat{\theta}}^{1} \frac{f(t) t}{F(t)} d t+\int_{\theta_{i}}^{1} \underbrace{F^{n-1}(t)(n-1) \frac{f(t) t}{F(t)}}_{=\left(F^{n-1}(t)\right)^{\prime} t} d t .
$$

Integrating the last term by parts, we get

$$
\begin{aligned}
\Pi\left(\hat{\theta}, \theta_{i}\right) & =F^{n-1}\left(\theta_{i}\right) \theta_{i}-(n-1) \int_{\hat{\theta}}^{1} \frac{f(t) t}{F(t)} d t+1-F^{n-1}\left(\theta_{i}\right) \theta_{i}-\int_{\theta_{i}}^{1} F^{n-1}(t) d t \\
& =1-(n-1) \int_{\hat{\theta}}^{1} \frac{f(t) t}{F(t)} d t-\int_{\theta_{i}}^{1} F^{n-1}(t) d t,
\end{aligned}
$$

which equals $\Pi\left(\hat{\theta}, \theta_{i}\right)$ in (8). Given this participation payoff, we determine participation behavior by taking into account the commonly known outside options. Since $\Pi\left(\hat{\theta}, \theta_{i}\right)$ is obviously strictly increasing in both, $\theta_{i}$ and $\hat{\theta}$, the minimum of $(8)$ is $\Pi(0,0)$ and the maximum is $\Pi(1,1)=1$. Therefore, we have full participation for $\bar{u} \leq \Pi(0,0)$, no participation for $\bar{u} \geq \Pi(1,1)=1$, and participation according to the cutoff value $\hat{\theta} \in(0,1)$ for $\Pi(0,0)<\bar{u}<\Pi(1,1)=1$. In the latter case, the cutoff is defined by $\bar{u}=\Pi(\hat{\theta}, \hat{\theta})$, the expected payoff of the indifferent bidder.

It remains to be shown that (14) does not only satisfy the first-order condition of $i$ 's best response problem but that it is indeed a maximiser for $\theta_{i}>\hat{\theta}$.

Similar to the above, consider $\theta, z \in(\hat{\theta}, 1]$. Start from (13) and insert (14) cancelling out $\alpha$ :

$$
\pi(z, \theta)=F^{n-1}(z)\left(\theta-(n-1) \int_{\hat{\theta}}^{z} \frac{f(k) k}{F(k)} d k\right)-\underbrace{\int_{z}^{1}(n-1) \int_{\hat{\theta}}^{s} \frac{f(k) k}{F(k)} d k\left(F^{n-1}(s)\right)^{\prime} d s}_{=:(A)} .
$$

Interchanging the order of integration, the term $(A)$ can be written as

$$
(A)=(n-1)(\int_{\hat{\theta}}^{z} \frac{f(k) k}{F(k)} \underbrace{\int_{z}^{1}\left(F^{n-1}(s)\right)^{\prime} d s}_{=1-F^{n-1}(z)} d k+\int_{z}^{1} \frac{f(k) k}{F(k)} \underbrace{\int_{k}^{1}\left(F^{n-1}(s)\right)^{\prime} d s}_{=1-F^{n-1}(k)} d k) .
$$


Now, reinsert into (20), cancel out the terms $\pm F^{n-1}(z)(n-1) \int_{\hat{\theta}}^{z} \frac{f(k) k}{F(k)} d k$, and get

$$
\begin{aligned}
\pi(z, \theta) & =\underbrace{F^{n-1}(z) \theta}_{=:(B)}-(n-1)\left(\int_{\hat{\theta}}^{z} \frac{f(k) k}{F(k)} d k+\int_{z}^{1} \frac{f(k) k}{F(k)}\left(1-F^{n-1}(k)\right) d k\right) \\
& =\underbrace{\theta-\int_{z}^{1}\left(F^{n-1}(k)\right)^{\prime} \theta d k}_{=(B)}-(n-1) \int_{\hat{\theta}}^{1} \frac{f(k) k}{F(k)} d k+\underbrace{\left.(n-1) \int_{z}^{1} \frac{f(k) k}{F(k)} F^{n-1}(k)\right) d k}_{\int_{z}^{1}\left(F^{n-1}(k)\right)^{\prime} k d k} \\
& =\theta-(n-1) \int_{\hat{\theta}}^{1} \frac{f(k) k}{F(k)} d k+\underbrace{\int_{z}^{1}\left(F^{n-1}(k)\right)^{\prime}(k-\theta) d k}_{=:(C)} .
\end{aligned}
$$

Note, that only $(C)$ depends on $z$. Thus,

$$
\pi(\theta, \theta)-\pi(z, \theta)=\int_{\theta}^{z}\left(F^{n-1}(k)\right)^{\prime}(k-\theta) d k .
$$

Since this is strictly positive for all $z \neq \theta$, where $z, \theta \in[0,1]$, this completes the proof.

Proof of Proposition 2. Obviously, if $\bar{u} \geq 1$, the outside option is superior to participation. Thus, we only need to consider $\bar{u} \in(-\infty, 1)$. We restrict attention to symmetric, pure-strategy equilibria with continuous, monotone bid functions. Therefore, participation is governed by a comparison of (candidate) equilibrium payoffs from participation, and the outside option. This implies a common cutoff valuation, denoted by $\hat{\theta} \in[0,1]$, above which a player participates in the auction. This includes the cases of 'full' and 'no' participation. Similar to the proof of Lemma 1, we can show that $\beta(\hat{\theta})=0$. The only difference is that, here, losing bidders' payoff is decreasing in (rather than independent of) the own bid.

Suppose that there is an equilibrium where player $i$ takes the outside option if $\theta_{i}<\hat{\theta}$ and otherwise participates and follows the common bid function $\beta$. Obviously, (11) is strictly increasing in $\theta_{i}$. Suppose that player $i$ 's rivals $j$ participate if $\theta_{j} \geq \hat{\theta}$, and if so, bid according to (11). W.I.o.g. we only consider bidder $i$ 's deviating bids $\beta(z)$ where $z \in[\hat{\theta}, 1]$ (and discuss outside options below). Recalling that $F^{(1: n-1)}=F^{n-1}$, bidder $i$ 's expected payoff is

$$
\begin{aligned}
\pi(z, \theta) & =F^{n-1}(z) \theta-\alpha \beta^{k}(z)-(n-1) \mathbb{E}\left[\alpha \beta(\Theta)^{k}\right], \\
\frac{\partial \pi(z, \theta)}{\partial z} & =\left(F^{n-1}(z)\right)^{\prime} \theta-\left(\alpha \beta^{k}(z)\right)^{\prime} .
\end{aligned}
$$

Applying the symmetric equilibrium condition, $z=\theta$, and the boundary condition $\beta(\hat{\theta})=0$ (see above), integration delivers (11). As a proof of sufficiency, we show that $\pi_{i}(\theta, \theta)>\pi_{i}(z, \theta)$ for all $z \in[\hat{\theta}, 1], z \neq \theta$. Note that the term $-\alpha(n-1) \mathbb{E}\left[\beta(\Theta)^{k}\right]$ cancels out in the first line of $(24)$ when we compute the difference $\pi_{i}(\theta, \theta)-\pi_{i}(z, \theta)$. Inserting the candidate, we get

$$
\begin{aligned}
\pi(\theta, \theta)-\pi(z, \theta) & =\theta\left(F^{n-1}(\theta)-F^{n-1}(z)\right)-\int_{z}^{\theta}\left(F^{n-1}(s)\right)^{\prime} s d s \\
& =\theta \int_{z}^{\theta}\left(F^{n-1}(s)\right)^{\prime} d s-\int_{z}^{\theta}\left(F^{n-1}(s)\right)^{\prime} s d s \\
& =\int_{z}^{\theta}\left(F^{n-1}(s)\right)^{\prime}(\theta-s) d s>0 .
\end{aligned}
$$


We continue by computing a participating bidder $i$ 's (candidate) equilibrium expected payoff, denoted by $\Pi\left(\hat{\theta}, \theta_{i}\right)$, i.e., we take the cutoff value $\hat{\theta}$ as given, and assume $\theta_{i} \geq \hat{\theta}$ and consider participating rivals' bids for $\theta_{j} \geq \hat{\theta}, j \neq i$. Inserting the candidate into the first line of (24), we get

$$
\Pi\left(\hat{\theta}, \theta_{i}\right)=\underbrace{F^{n-1}\left(\theta_{i}\right) \theta_{i}-\int_{\hat{\theta}}^{\theta_{i}}\left(F^{n-1}(s)\right)^{\prime} s d s}_{=:(A)}-(n-1) \underbrace{\int_{0}^{1} \int_{\hat{\theta}}^{\theta_{j} \geq \hat{\theta}}\left(F^{n-1}(s)\right)^{\prime} s d s f\left(\theta_{j}\right) d \theta_{j}}_{=:(B)}
$$

Using integration by parts, term $(A)$ in the above can be written as

$$
(A)=F^{n-1}\left(\theta_{i}\right) \theta_{i}-\left(\left[F^{n-1}(s) s\right]_{\hat{\theta}}^{\theta_{i}}-\int_{\hat{\theta}}^{\theta_{i}} F^{n-1}(s) d s\right)=F^{n-1}(\hat{\theta}) \hat{\theta}+\int_{\hat{\theta}}^{\theta_{i}} F^{n-1}(s) d s .
$$

Interchanging the order of integration, term $(B)$ can be written as

$$
(B)=\int_{\hat{\theta}}^{1} \int_{s}^{1} f\left(\theta_{j}\right) d \theta_{j}\left(F^{n-1}(s)\right)^{\prime} s d s=\int_{\hat{\theta}}^{1}(1-F(s))\left(F^{n-1}(s)\right)^{\prime} s d s .
$$

Reinserting these expressions for $(A)$ and $(B)$ gives (10).

Finally, given this participation payoff, we determine participation behavior by taking into account the value of the commonly known outside options. Note that (10) is strictly increasing in both, $\theta_{i}$ and $\hat{\theta}$. The former is obvious and the latter can be seen in the first derivative,

$$
\frac{\partial \Pi\left(\hat{\theta}, \theta_{i}\right)}{\partial \hat{\theta}}=\left(F^{n-1}(\hat{\theta})\right)^{\prime} \hat{\theta}+F^{n-1}(\hat{\theta})-F^{n-1}(\hat{\theta})+(n-1)(1-F(\hat{\theta}))\left(F^{n-1}(\hat{\theta})\right)^{\prime} \hat{\theta},
$$

where the second and third term cancel out, while the remaining terms are positive. Thus, for $\hat{\theta}, \theta_{i} \in[0,1]$ and $\theta_{i} \geq \hat{\theta}$, the maximum of $(10)$ is $\Pi(1,1)=1$ while the minimum is $\Pi(0,0)$. Therefore, we have full participation for $\bar{u} \leq \Pi(0,0)$, and participation according to the cutoff value $\hat{\theta} \in(0,1)$ whenever $\Pi(0,0)<\bar{u}<\Pi(1,1)=1$. In the latter case, the cutoff is defined by $\bar{u}=\Pi(\hat{\theta}, \hat{\theta})$, the payoff of the indifferent bidder.

\section{References}

Amann, E., And W. Leininger (1996): "Asymmetric All-Pay Auctions with Incomplete Information: The Two-Player Case," Games and Economic Behavior, 14, 1-18.

Baye, M. R., D. Kovenock, and C. De VRIEs (1993): "Rigging the Lobbying Process: An Application of All-Pay Auctions," American Economic Review, 83, 289-94.

(2005): "Comparative Analysis of Litigation Systems: An Auction-Theoretic Approach," The Economic Journal, 115(505).

(2012): "Contests with rank-order spillovers," Economic Theory, 51(2), 315-50.

Bulow, J., And P. Klemperer (1999): "The Generalized War of Attrition," American Economic Review, 89(1), 175-189. 
Clark, D. J., and C. Rits (1998): "Competition over More than One Prize," American Economic Review, 88(1), 276-89.

Congleton, R. D., A. L. Hillman, and K. Konrad (eds.) (2007): 40 Years of Rent Seeking Research. Springer, Heidelberg.

Corchón, L. C. (2007): "The theory of contests: a survey," Review of Economic Design, 11, 69-100.

Dasgupta, P. (1986): "The Theory of Technological Competition," in New Developments in the Analysis of Market Structure, ed. by J. E. Stiglitz, and G. F. Mathewson, pp. 519-49. MIT Press, Cambridge, Mass.

Fibich, G., A. Gavious, and A. Sela (2006): "All-pay auctions with risk-averse players," International Journal of Game Theory, 34, 583-99.

Garfinkel, M. R., and S. Skaperdas (2006): "Economics of Conflict: An Overview," in Handbook of Defense Economics, ed. by T. Sandler, and K. Hartley, vol. 2, chap. 3. Elsevier.

Geradin, D. (2010): "Reverse Hold-ups: The (Often lgnored) Risks Faced by Innovators in Standardized Area," in The Pros and Cons of Standard Setting, ed. by Konkurrensverket, Swedish Competition Authority, chap. 6, pp. 101-43.

Goeree, J., And J. Turner (2000): "All-pay-all auctions," University of Virginia, Mimeo.

Goeree, J. K., E. Maasland, S. Onderstal, and J. L. Turner (2005): "How (Not) to Raise Money," Journal of Political Economy, 113, 897-926.

Güth, W., and E. Van Damme (1986): "A Comparison of Pricing Rules for Auctions and for Division Games," Social Choice and Welfare, 3, 177-98.

Hillman, A. L., and J. G. Riley (1989): "Politically contestable rents and transfers," Economics and Politics, 1, 17-39.

Hillman, A. L., and D. Samet (1987): "Dissipation of contestable rents by small numbers of contenders," Public Choice, 54, 63-82.

Hopkins, E., and T. Kornienko (2007): "Cross and Double Cross: Comparative Statics in First Price and All Pay Auctions," B.E. Journal of Theoretical Economics, 7, Article 19.

Kaplan, T., I. Luski, A. Sela, and D. Wettstein (2002): "All-Pay Auctions with Variable Rewards," Journal of Industrial Economics, 50(4), 417-30.

Klose, B., And D. Kovenock (2011a): "The All-Pay Auction with Complete Information and Identity-Dependent Externalities," University of Zurich, Discussion paper, 73. 2011.

Konrad, K. (2008): Strategy and Dynamics in Contests. Oxford University Press, Oxford.

Krishna, V., And J. Morgan (1997): "An Analysis of the War of Attrition and the All-Pay Auction," Journal of Economic Theory, 70(2), 343-62.

Lizzeri, A., AND N. Persico (2000): "Uniqueness and existence of equilibrium in auctions with a reserve price," Games and Economic Behavior, 30(1), 83-114. 
MaAsland, E., and S. Onderstal (2007): "Auctions with Financial Externalities," Economic Theory, 32, 551-74.

Moldovanu, B., And A. Sela (2001): "The Optimal Allocation of Prizes in Contests," American Economic Review, 91(3), 542-58.

Organisation for Economic Co-operation and Development (2010): "Working Party No. 2 on Competition and Regulation: Standard Setting (United States)," Working Paper, DAF/COMP/WP2/WD(2010)28.

Parreiras, S. O., And A. Rubinchik (2010): "Contests with three or more heterogeneous agents," Games and Economic Behavior, 68(2), 703-715.

Szech, N. (2011): "Asymmetric All-Pay Auctions with Two Types," University of Bonn, Discussion paper, January. 